\title{
OFICINAS DE LA MUJER EN MUNICIPIOS COSTARRICENSES: ¿GARANTÍA FORMAL O REAL?
}

WOMEN'S OFFICES IN COSTA RICAN MUNICIPALITIES: FORMAL OR REAL GUARANTEE?

\author{
Joselyn Vargas Mayorga \\ jos.vargas.mayorga@gmail.com \\ https://orcid.org/0000-0003-3228-5410 \\ Gonzalo Calvo Bonilla \\ goncabo18@gmail.com \\ https://orcid.org/0000-0002-6454-1039
}

\section{RESUMEN}

En Costa Rica, aun cuando 65 de los 82 cantones que conforman el territorio nacional cuentan con una Oficina de la Mujer, muchas no tienen funcionarios ni presupuesto asignados, y pocas de las que sí cuentan con recursos han honrado el espíritu de la ley que promovió su creación. En el artículo se señalan los casos de algunos de los municipios que enfrentan retos más apremiantes y se rescatan experiencias valiosas dignas de ejemplo. Los autores concluyen que una aplicación efectiva de la ley requiere de la capacitación de funcionarios municipales respecto de la importancia de entornos públicos más diversos e inclusivos, de modo que haya un control constante de los avances y los retrocesos en la materia.

\section{PALABRAS CLAVE}

Costa Rica, gobierno local, equidad de género, políticas públicas

\section{Escuela de Ciencias Políticas \\ Universidad de Costa Rica}

Costa Rica

\section{ABSTRACT}

In Costa Rica, even though 65 of the 82 cantons that make up the national territory have a Women's Offices, many of them don't have officials or budgets assigned, and few of those who do have resources have honored the spirit of the law that promoted their creation. The article identifies the cases of some of the municipalities that face more pressing challenges and rescued valuable experiences worthy of mention. The authors conclude that an effective application of the law involve the training of municipal officials regarding the importance of more diverse and inclusive public environments, with the objective of having a constant control of the advances and setbacks in the matter.

\section{KEYWORDS}

Costa Rica, local government, gender equality, public policies 


\section{OFICINAS DE LA MUJER EN MUNICIPIOS COSTARRICENSES: ¿GARANTIIA FORMAL O REAL?}

Por Joselyn Vargas Mayorga y Gonzalo Calvo Bonilla

Como parte de un contexto universal permeado por luchas de reivindicación a cargo de movimientos feministas, aunado al consenso de la Organización de las Naciones Unidas y a sus Conferencias sobre la Mujer, el Estado costarricense ha adquirido desde la década de los setenta mayores compromisos tendientes al cumplimiento efectivo de los derechos de las mujeres. De esta manera, en 1974, se creó la Oficina de Programas para la Mujer y la Familia, instancia adscripta al Ministerio de Cultura, Juventud y Deportes que se encargó de coordinar acciones relativas a la Primera Conferencia Mundial de la Mujer, realizada en México (1975). 
Una década después, esta oficina se convirtió en el Centro Nacional para el Desarrollo de la Mujer y la Familia, «ente rector de políticas nacionales a favor de lasmujeres, conpersoneríajurídica ypatrimoniopropio»(InstitutoNacionaldelas Mujeres, en línea). Sin embargo, no fue hasta 1998, con la promulgación de la Ley de la República 7.801, ${ }^{1}$ que este Centro - denominado a partir de entonces Instituto Nacional de las Mujeres - adquirió carácter de entidad autónoma y descentralizada. Precisamente, es en el seno de esta Ley que se enmarca la promoción de las Oficinas de la Mujer, como medio para garantizar, para coordinar y para ejecutar esfuerzos que permitan la igualdad real de oportunidades entre hombres y mujeres.

A pesar de esto, si bien la Ley enfatiza la apremiante tarea de democratizar el espacio público local no establece ni los mecanismos ni los medios, ni los presupuestos destinados a esta labor. $Y$ es que, acorde a nuestro ordenamiento jurídico, el legislador no puede inmiscuirse en estos territorios, por cuanto las municipalidades cuentan con autonomía normativa y administrativa, siendo que la primera le da la libertad de dictar ordenamiento propio en materias de su competencia (organización y servicios), mientras que la segunda le concede capacidad para autoadministrarse a su conveniencia. Tampoco puede establecer partidas o cuotas mínimas de ejecución, por cuanto es competencia exclusiva del órgano municipal dictar su propio presupuesto y, por ende, dotar (o no) de músculo a sus políticas públicas.

La autonomía es tal que la municipalidad no tiene la obligación en medida estricta de incorporar dentro de su estructura organizativa a esta oficina, ${ }^{2}$ por cuanto el legislador solo puede tutelar los fines que se derivan del Derecho Constitucional, mas no los medios, lo que resulta en que «el Estado o sus instituciones, a lo sumo, lo único que pueden hacer es sugerir o promover este tipo 
de órganos, nunca imponérselos a los municipios, aunque tal acto normativo sea el resultado del uso de la potestad de legislar» (Contraloría General de la República, 2003, en línea).

En cualquier caso, 65 de los 82 cantones tienen adscrita a su organización una Oficina de la Mujer y Equidad de Género. No obstante, en muchos de los casos no existe personal a cargo y mucho menos presupuesto asignado para sus planes operativos. En otros, sus labores se han enfocado a la realización de ferias de empleo «abiertas al público», sin ningún tipo de acompañamiento o de asesoría, y que derivan en formas de discriminación indirectas al establecer condiciones de acceso que ignoran la disponibilidad de tiempo de las mujeres dados los roles de género reproductivos (esposas, madres y cuidadoras) que tradicionalmente han tenido que asumir. También existen aquellas que se han convertido en centros de «recepción» de las denuncias por violencia de género, pero que realmente no les dan seguimiento. Muy pocos han sido los municipios que han honrado el espíritu de la Ley 7.801 que les dio origen, al ejercer un papel determinante en el cuestionamiento de estereotipos y de prejuicios que invisibilizan el aporte de la mujer y la confinan al área de lo «privado».

Dicho esto, nos preguntamos: ¿Qué esfuerzos ya implementados deben rescatarse como modelo para las demás comunidades y cuáles deben mejorarse? Y, sobre todo, ¿qué desafíos enfrentamos con miras a la equiparación de oportunidades y a la incorporación efectiva de las mujeres en la vida pública (productiva y política) de nuestras comunidades? 


\section{EXPERIENCIAS A RESCATAR}

Como órgano descentralizado, la municipalidad adquiere principal importancia porque es el primer contacto que tiene la ciudadanía con el fenómeno del poder. A través de ella, la población hace eco de sus necesidades y de sus demandas para que estas sean incorporadas en la formulación de políticas públicas cantonales que mejoren la calidad de su diario vivir. Este apartado da cuenta de tres casos recientes que han logrado incorporar acciones correctivas al patriarcado ${ }^{3}$ sintomático que rodea a la sociedad costarricense, tanto en el Gran Área Metropolitana como en las periferias y en los territorios más alejados.

A destacar se encuentra la Municipalidad de Escazú, que en 2013 fue pionera con la iniciativa que creó la «Escuela de Formación en Liderazgo y Ciudadanía para Mujeres», con el objetivo de que se constituya en un vehículo para el empoderamiento y la incorporación efectiva en el mercado laboral. ${ }^{4}$ Además del fortalecimiento de habilidades emprendedoras, este proyecto brinda capacitaciones especializadas en derechos humanos y en igualdad de género para los hombres del cantón, con el objetivo de que estos tomen conciencia del importante rol de la mujer en la sociedad y de que sean facilitadores del reclamo sobre el papel al que tienen derecho (Gamboa, 2016, en línea).

Otro caso a destacar es el de la Municipalidad de Heredia, que brinda un servicio pormenorizado en diversas ramas de acción. Además del acompañamiento a mujeres sobrevivientes de violencia doméstica, el municipio ofrece actividades recreativas, como "Apropiación del cuerpo por medio de la danza", y fomenta el emprendedurismo mediante ferias de comercialización, capacitación y orientación cantonal. Con el fin de asumir un rol preponderante en la 
deconstrucción de individuos y en el derribo de estereotipos, junto con el Instituto de Masculinidad, Pareja y Sexualidad ofrece un espacio terapéutico «para hombres con problemas de poder y de control» (Oficina de Igualdad y Equidad de Género [OIEG], en línea). Asimismo, ha implementado en centros colegiales el «Proyecto de Vida Adolescente», que se enmarca en la Política de Igualdad y Equidad, y que pretende abordar la «deconstrucción de mitos y de estereotipos de género, los mandatos de maternidad y de paternidad, y la respuesta sexual humana y actividad sexual temprana en la adolescencia» (OIEG, en línea).

Por último, pero ciertamente no menos importante, es la labor de la Municipalidad de Mora, que brinda atención a mujeres indígenas que, dada su condición, enfrentan discriminación múltiple. Esta particularidad también se evidencia en otros países de la región centroamericana, y de forma particular en Guatemala, donde

[...] por razones histórico-culturales, sociales, económicas y políticas, las mujeres indígenas enfrentan una triple condición de discriminación, de opresión y de exclusión: por ser mujeres, por ser indígenas, y porque la mayoría vive en condiciones de pobreza; lo que compromete un esfuerzo especial por parte de las OMM para garantizar que esta situación sea identificada y visibilizada en las acciones institucionales de la municipalidad (Oficina Municipal de la Mujer, 2008, p. 25). 
De tomar en cuenta estas realidades, nacen proyectos como Lideres Comunales, el programa Mujer y Microempresa, la «Red para la prevención y atención de la violencia intrafamiliar" y alternativas laborales, todos ellos abordados desde una perspectiva de la diversidad y de la interculturalidad, y orientados a contribuir con la generación de habilidades, el fortalecimiento educativo, el acceso a las tecnologías de información, el emprendedurismo y la formación humana (Oficina de la Mujer, Municipalidad de Mora, en línea).

\section{MUNICIPALIDADES CON RETOS IMPERIOSOS DE ATENDER}

Como se indicó anteriormente, en Costa Rica solo 65 de las 82 municipalidades cumplen con la obligación legal de contar con una oficina dedicada a promover acciones dedicadas a la defensa de los derechos de las mujeres. De esas 65 oficinas, cinco se encuentran cerradas temporalmente y únicamente trece cuentan con la dirección de profesionales especializadas en género.

En cuanto a las municipalidades en estado crítico respecto de la ejecución de las Oficinas de la Mujer, es de destacar el caso del cantón de San José (por mucho el municipio con mayor poderío económico del país), que ni siquiera incluye información relacionada con esta oficina. La única fuente que la menciona es la página web del Sistema de Información Cultural de Costa Rica (SICUltura), ${ }^{5}$ que lo hace al indicar el lugar en el cual se debe realizar el registro para participar del Servicio de Intermediaciòn de Empleo que el cantón ofrece a sus habitantes. 
Si bien el desempleo es uno de los retos principales para lograr la igualdad entre mujeres y hombres, ${ }^{6}$ el objetivo descrito en la página del sicultura no responde a los fines que la Ley le asigna a esta oficina, ni mucho menos a lo que se esperaría de los productos de políticas públicas dedicadas a eliminar la discriminación contra las mujeres. Aunado a lo anterior, resalta el poco interés de la municipalidad por informar adecuadamente a las ciudadanas sobre las labores de esta oficina, más si se considera que cuenta con una página web moderna y actualizada.

Preocupa, también, la gestión de la Municipalidad de Cartago, una de las provincias del Valle Central con mayor cantidad de recursos debido las actividades económicas concentradas en el cantón y a la cantidad de habitantes que aportan con sus impuestos al desarrollo local. Al igual que la Municipalidad de San José, la de Cartago no cuenta en su página web con información dedicada a dar a conocer las labores de la Oficina de la Mujer, que únicamente aparece mencionada en el sitio del sicultura que le asigna a dicha oficina el objetivo general de la función municipal de este cantón (sicultura, en línea).

En el caso particular de esta municipalidad, los investigadores intentaron en numerosas ocasiones acceder a través de mecanismos formales (llamadas telefónicas y correos electrónicos) y de mecanismos informales (a través de las gestiones de un funcionario municipal) a conversar con el personal a cargo de la Oficina; sin embargo, después de semanas no fue posible, ya que la funcionaria responsable de la Oficina tiene a su cargo, también, toda el área social (participación ciudadana, becas, salud, vivienda, etc.). 
Lo anterior deja entrever la ausencia (por falta de voluntad política) de recursos destinados a la atención de las mujeres del cantón, lo que lleva a preguntarse por las posibilidades que estas tienen para contar con una respuesta rápida a sus problemas. ¿Qué pasa si una mujer necesita asesoría inmediata para interponer una denuncia por violencia doméstica?, ¿qué posibilidades tendrá una mujer para proponer soluciones institucionales a sus problemas si el órgano encargado no escucha su voz?

La falta de interés en destinar recursos a los problemas particulares que aquejan a las mujeres no es novedad. Le conviene al sistema patriarcal y capitalista perpetuar sus condiciones de vulnerabilidad. La ausencia de atención pronta y cumplida impide atender adecuadamente problemas tan serios como la violencia contra las mujeres y la discriminación en todos los ámbitos. No debe darse por menos el hecho de que estas oficinas no cumplan con sus objetivos, ya que ponen en riesgo la vida misma de las mujeres.

\section{REFLEXIONES FINALES}

Si bien Costa Rica ha hecho esfuerzos importantes para eliminar la discriminación contra las mujeres, estos han sido insuficientes. Prueba de ello es la ausencia de mecanismos en los gobiernos locales, que se supone son los más cercanos a la ciudadanía para atender esta problemática.

La lucha de las mujeres por el reconocimiento de sus derechos es de larga data; sin embargo, es evidente que tiene todavía un largo camino por recorrer. En el caso particular de los gobiernos locales en Costa Rica, o no se las toma en cuenta del todo o se sigue concibiendo a las mujeres como madres, 
esposas, amas de casa, beneficiarias pasivas, grupos vulnerables y clientelas electorales; y no, precisamente, como sujetos de derecho y como personas activas en las dinámicas comunales.

Existe una vasta literatura con respecto a la incorporación de la perspectiva de género en la administración pública. Sin embargo, pocas contemplan la importancia de incorporar esta perspectiva en gobiernos locales. Una de ellas es la Guía para la Equidad de Género en el Municipio (Grupo Interdisciplinario sobre Mujer, Trabajo y Pobreza [GIMTRAP], 2004), que propone principios y métodos que deberían aplicarse para implementar la gestión municipal con equidad de género, la cual requiere de

- Incorporar procesos incluyentes y participativos, individuales y colectivos, que integren a la ciudadanía en todas sus expresiones sociales e institucionales.

Construir alianzas entre diferentes actores de la sociedad civil, del gobierno municipal, del ámbito estatal y nacional, para articular objetivos y acciones hacia el logro de la equidad y de la igualdad de oportunidades en los ámbitos municipales.

Promover y fortalecer la formación de redes sociales, como estrategia de difusión y como toma de conciencia de la equidad como valor del desarrollo humano.

Generar sistemas de información diferenciados para el análisis de género y conocer de manera objetiva las condiciones y las posiciones de las mujeres en el municipio. 
Generar y mejorar los sistemas de evaluación de la gestión, considerando los objetivos logrados y propuestos en el marco de la equidad de género.

- Hacer la gestión más transparente, utilizando instrumentos de rendición de cuentas que permitan mostrar las decisiones y las acciones de equidad de género (GIMTRAP, 2004, p. 17).

Al reflexionar sobre los puntos anteriores, es evidente que si bien las Oficinas de la Mujer en los gobiernos locales son un esfuerzo destacable resultan insuficientes para la atención de las mujeres. No solo porque en ocasiones no existen o no cumplen con funciones relacionadas con el fin para el que fueron creadas, sino porque continúan concibiendo a las mujeres como sujetas pasivas y no consideran sus aportes dentro de las dinámicas comunales.

Para que realmente existan municipalidades con perspectiva de género, se hace necesario capacitar a los funcionarios y a las funcionarias municipales respecto de la importancia de incluir a las mujeres en las acciones de los planes de gobierno de las municipalidades, comprender que las mujeres conforman el 50\% de la población y que lo lógico es que sean parte activa de la toma de decisiones y receptoras de los programas municipales.

Asimismo, y debido a que el Poder Ejecutivo no tiene injerencia en los gobiernos locales, es importante la coordinación de acciones interinstitucionales a través de organizaciones como la Unión Nacional de Gobiernos Locales (UNGL) $)^{6} \mathrm{u}$ otras, que se encarguen de coordinar acciones concretas que impliquen desde la educación de los funcionarios y las funcionarias municipales hasta la elaboración de programas en conjunto para eliminar la discriminación contra las mujeres. 


\section{REFERENCIAS ELECTRÓNICAS}

Contraloría General de la República de Costa Rica (2003). Opinión Jurídica No. O.J.018-2003. Recuperado de http://www.pgrweb.go.cr/scij/Busqueda/Normativa/pronunciamiento/pro_ficha.aspx?param1=PRD\&param6=1\&nDictamen=11654\&strTi$\mathrm{pM}=\mathrm{T}$

Gamboa, D. (31 de octubre de 2016). Primera escuela de empoderamiento de mujeres gradúa a más de 500 ticas. Perfil. Recuperado de http://www.revistaperfil.com/pb/vida/cultura/primera-escuela-de-empoderamiento-de-mujeres-gradua-a-mas-de-500-ticas/7BA4ZNUFXZDVJPU55YFX6E6054/story/

Grupo Interdisciplinario sobre Mujer, Trabajo y Pobreza (GIMTRAP) (2004). Guía para la Equidad de Género en el Municipio. Recuperado de http://www.redmujer.org. ar/articulos/art_11.pdf

Instituto Nacional de las Mujeres (INAMU). Nuestra Historia. Recuperado de http:// www.inamu.go.cr/asi-somos

JASS Mesoamérica (2012). Diccionario de la Transgresión Feminista. Recuperado de repositorio.ciem.ucr.ac.cr/bitstream/123456789/60/1/RCIEM046.pdf

Ley 7.794, Costa Rica (1998). Código Municipal de Costa Rica. Recuperado de www.tse. go.cr/pdf/normativa/codigomunicipal.pdf

Ley 7.801, Costa Rica (1998). Creación del Instituto Nacional de las Mujeres (INAMU). Recuperado de http://extwprlegs1.fao.org/docs/texts/cos135890.doc

Oficinas de la Mujer en municipios costarricenses: 
Municipalidad de Cartago (2017). Oficina de la Mujer. Recuperado de https://si.cultura.cr/capacitacion/municipalidad-de-cartago-oficina-equidad-de-genero-y-desarrollo-social.html

Municipalidad de San José (2014). Oficina de la Mujer. Recuperado de: https://si.cultura.cr/capacitacion/municipalidad-de-san-jose-oficina-de-la-mujer.html

Oficina de lgualdad y Equidad de Género (OIEG), Municipalidad de Heredia. Recuperado de https://www.heredia.go.cr/es/social-wellness/office-of-gender/programs-researches/\%C3\%A1reas-de-trabajo-de-oficina-de-igualdad-equidad

Oficina de la Mujer, Municipalidad de Mora. Recuperado de http://visitemora.com/ visitemora/15-areas-gov/44-ofimujer

Oficina Municipal de la Mujer (OMM) (2008). Manual de Funciones de la Oficina Municipal de la Mujer. Un componente para la participación ciudadana y social con enfoque de género y juventud. Ciudad de Guatemala, Guatemala: Fundación Guillermo Toriello. Recuperado de http://eeas.europa.eu/archives/delegations/guatemala/documents/more_info/virtual_library/manual_omm_es.pdf

Sistema de Información Cultural de Costa Rica (SICULTURA). Recuperado de https:// si.cultura.cr/ 
Con X

\section{NOTAS}

1 «Art. 4. Atribuciones: Para el cumplimiento de sus fines, el Instituto tendrá las siguientes atribuciones: [...] e) Promover la creación de oficinas ministeriales, sectoriales y municipales de la mujer; además, garantizar y coordinar su funcionamiento» (Ley 7.801, en línea).

2 En lo que nos ocupa, el Código Municipal - como parte del Ordenamiento Jurídico Administrativo Costarricense, de acatamiento obligatorio y atinente al quehacer municipal a nivel nacional- especifica en su artículo 4, inciso i, como una de sus atribuciones: «lmpulsar políticas públicas locales para la promoción de los derechos y la ciudadanía de las mujeres, en favor de la igualdad y la equidad de género», para lo cual crea en su artículo 49 una Comisión Permanente de la Condición de la Mujer para darle seguimiento a estas iniciativas (Ley 7.794, en línea). No debe pasarse por alto que, residualmente, estos órganos también promueven acciones en beneficio del fortalecimiento de la familia.

3 El patriarcado hace referencia a un sistema jerárquico de valores que deriva en una desigualdad en el acceso al poder. Si bien no es un concepto que nace de las teorías feministas ha sido ampliamente revisado y expandido por estas, al considerarlo como la manifestación y la institucionalización del dominio de lo masculino sobre lo femenino (JASS Mesoamérica, 2012).

4 Más información en https://www.escazu.go.cr/es/noticias/escuela-de-formacion-en-liderazgo-y-ciudadania-para-mujeres

5 El Sistema de Información Cultural de Costa Rica (sicultura) es uno de los componentes de la Unidad de Cultura y Economía (UCE) del Ministerio de Cultura y Juventud, y «pone a disposición de artistas y del público en general diversos servicios e información cultural actualizada y útil para la definición de políticas y para la toma de decisiones tanto desde el ámbito público como 
privado» (sICUltura, 2014, en línea). Es debido al objetivo de brindar información actualizada que el SiculturA destinó una página web para las 81 municipalidades en Costa Rica. Sin embargo, muchas municipalidades no mantienen actualizada su información en este Sistema.

6 Según datos de la «Encuesta Continua de Empleo: Indicadores del Mercado Laboral Costarricense CUARTO TRIMESTRE 2016», elaborada por el Instituto Nacional de Estadística y Censos, el desempleo en las mujeres es del 13,8\%, el doble del presentado en hombres (6,9\%).

7 La Unión Nacional de Gobiernos Locales (UNGL), con casi cuarenta años de existencia, fue fundada en 1977 ante la necesidad de las municipalidades de contar con una organización que las uniera para impulsar la descentralización política y administrativa del Estado costarricense, y para fortalecer a los gobiernos locales mediante políticas y normas que amplíen su autonomía, sus competencias y sus recursos. Su misión es ser la entidad política y gremial (expresión legítima del Régimen Municipal) que representa, que posiciona y que defiende a las municipalidades; y que fortalece la autonomía política, administrativa, financiera e institucional de dinámicos gobiernos locales, promotores del desarrollo social, humano y sostenible de sus territorios. 